\title{
Design Framework for a Simple Robotic Ankle Evaluation and Rehabilitation Device
}

\author{
Christos E. Syrseloudis, Ioannis Z. Emiris, Constantinos N. Maganaris and Theodoros E. Lilas
}

\begin{abstract}
This paper juxtaposes simple yet sufficiently general robotic mechanisms for ankle function evaluation, measurement and physiotherapy. For the choice, design and operation of the mechanism, a kinematics model of foot is adopted from biomechanics, based on the hypothesis that foot kinematics are similar to a $2 \mathrm{R}$ serial robot. We undertake experiments, using a 3D scanner and an inertial sensor in order to fully specify the design framework by studying a larger sample of healthy subjects. Our experimental analysis confirms and enhances the $2 R$ foot model, and leads us to the choice of the specific mechanism. We compute the required workspace and thus address the issues required for a complete and efficient design. The robot must be capable to perform several multi-axis motions and sustain a significant range of forces and torques. We compare mechanisms based on serial and parallel robots, and choose a parallel tripod with an extra rotation axis for its simplicity, accuracy and generality.
\end{abstract}

\section{INTRODUCTION}

Ankle injuries are very common and they often require rehabilitation. The equipment used for ankle rehabilitation is usually simple, comprising elastic bands, foam rollers and wobble boards [19]. Such rehabilitation devices are typically used in regimens that include exercises both in the clinic and at home. Nevertheless, these devices lack quantitative diagnostic capabilities to allow therapists to monitor the patient's progress. More importantly, they cannot be used for assessment, hence the interest for an automatic device for complete physiotherapy and evaluation.

Several mechanisms have been proposed to help the physiotherapist [1]. Quite successful is the work at Rutgers University, including a haptic interface for ankle and hand rehabilitation. For ankle rehabilitation, a haptic interface was developed by G. Burdea et al. [5], based on a Stewart platform that applies variable forces and virtual reality exercises on the foot, including remote control operation. However, its 6 dof structure is rather redundant and there is no report for the design criteria; we aim at a smaller and more economic alternative, easier to move and operate (e.g. noise can be an issue with large devices). We also emphasize adaptation to the specific characteristics of each patient's foot.

In [7], the study of ankle injuries and ankle functional anatomy was based on an orientation image space; 3

C.E. Syrseloudis, I.Z. Emiris are with the Department of Informatics and Telecommunications, National University of Athens, GREECE c.sirsel@di.uoa.gr

C.N. Maganaris is with the Institute for Biomedical Research into Human Movement and Health (IRM), Manchester Metropolitan University, UK c.n.maganaris@mmu.ac.uk

T.E Lilas is with the Department of Shipping Trade and Transport, University of the Aegean, GREECE lilas daegean.gr platform-type ankle rehabilitation mechanisms were considered and their mobility, stiffness and constraints analyzed. But other design criteria are not analyzed nor is the construction of such platforms.

Parallel robot design concerns the geometric parameters calculation of a parallel robot with specific architecture satisfying several criteria. A method for parallel robot design is based on interval analysis as it is proposed in [11]. This involves multidesign criteria (workspace covering, accuracy bounds satisfaction, stiffness etc).

\section{A. Our contribution}

We focus on the design framework of a simple yet sufficiently general robot for ankle injuries treatment. The allowable motions of foot are described and the foot trajectories during physiotherapy exercises are defined. Previous works were based on approximate estimations for the range of foot motions and they make no reference for the allowable movements. Here we use a foot kinematics model, based on the biomechanics literature. We show that not all movements of the ankle joint are allowable, since the rotation axes in the ankle have a specific position and the trajectories of the foot are constrained.

By using an existing kinematics model of the foot we roughly compute the translation and orientation workspace of the robot. Extensive experiments with a 3D scanner and an inertial sensor have been performed to fully determine the kinematics behavior of the foot. The data obtained define the translation and orientation workspaces, and the velocity and acceleration ranges. The manipulator must have the ability to follow the basic human foot motions in the plantarflexiondorsiflexion and inversion- eversion directions with the upper leg fixed, and sustain a significant range of forces and torques. We compare serial and parallel robots, and choose a parallel tripod with an extra rotation axis for its modularity, simplicity, accuracy and generality. Moreover, its workspace answers the specifications obtained from our experiments (which confirm the available biomechanics models).

\section{ANKLE MOTION DESCRIPTION}

The human ankle has a complex multi-joint structure. The central bone is the talus. Its surrounding bones are the calcaneus, the navicular and the cuboid; they are responsible for the rotation of the ankle joint in 3D. The upper part of the talus articulates with the shank segment through the tibia and fibula bones. This is the upper ankle joint (UAJ); it supports the rotational dorsiflexion/plantarflexion motion. The movements between the fore bones are strictly coupled. 


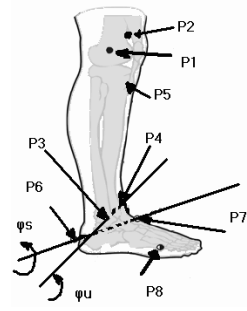

Fig. 1. Point marks on the foot and ankle's main rotation axes.

Motion of the foot wrt the talus is regarded as a rotation about the (fixed) subtalar axis (STJ); this supports the rotation supination/pronation, which has an inversion/eversion and an abduction/adduction component.

We adopt an ankle kinematics model from biomechanics [9]. The lower limb is assumed to be composed of 3 rigid links capable to rotate between each other: the shank, the talus and the foot configuring a serial manipulator. The main movements of the foot are the plantarflexion/dorsiflexion and inversion/eversion. The size of foot bones and their relative positions as well as the orientation of rotation axes determine the foot kinematics. Many factors influence the joint rotation, e.g. shape of articular surfaces, position of rotation axes. Constraint and resistance on the foot motions are due to ligaments, capsules and tendons.

The parameters of this model are specified by a number of point markers that have been assigned on the human foot as in fig. 1. These point markers are used to obtain a set of distance measurements. We assign frame $O_{1}$ at the knee, centered between $P_{1}, P_{2}$, with the $z$-axis parallel to $\left(P_{1}, P_{2}\right)$ and the $x$-axis vertical, passing through the midpoint of $\left(P_{3}, P_{4}\right)$. By using the Denavit-Hartenberg(D-H) method we assign relative frames $O_{i}$ between the moving links. $T_{i}^{i+1}$ is the transformation matrix from $O_{i}^{i+1}$ into $O_{i}$.

The transformation matrix from the last into the first coordinate system is given from the relationship $T_{1}^{3}=T_{1}^{2} T_{2}^{3}$. For a point $P=\left[\begin{array}{llll}x & y & z & 1\end{array}\right]^{T}$ on the last(foot) coordinate system the above transformation into the first(shank) coordinate system can be expressed as $P_{o}=\left[\begin{array}{llll}x_{o} & y_{o} & z_{o} & 1\end{array}\right]^{T}$ :

$$
P_{o}=T_{1}^{3} P
$$

from which the coordinates $x_{o}, y_{o}, z_{o}$ are nonlinear functions $f_{i}\left(a_{i}, \alpha_{i}, d_{i}, \vartheta_{i}, x, y, z\right)$ of the D-H parameters $a_{i}, \alpha_{i}, d_{i}, \vartheta_{i}$. These equations give a parametric formula in the movement of $P$ wrt the fixed coordinate system of the shank. The independent variables of the model are angles $\vartheta_{2}$ (dorsiflexion/plantarflexion), $\vartheta_{3}$ (inversion/eversion) while $\vartheta_{1}$ is constant. According to the right-hand coordinate system assigned to the lower limb, the signs for rotation angles are: dorsiflexion(+), plantarflexion(-), eversion(+) and inversion $(-)$. Movements of the left leg are assumed to be the mirror-image of the right leg [9]. The parameters $\alpha_{i}, a_{i}, d_{i}$ depend on the foot anatomy and size.

The transformation matrices were estimated for a male subject, and distances between the bony landmarks taken from [9]. From these data, a kinematics model of the foot

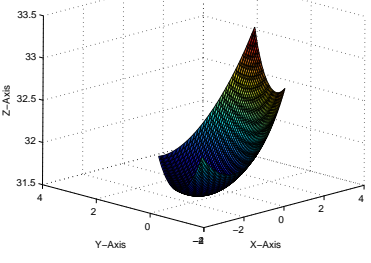

(a) Translation

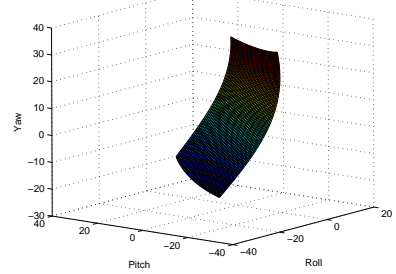

(b) Rotation
Fig. 2. Workspace from a sole-point under the ankle.

was based on homogenous matrix transformations in Euler angles. Using the Maple9.5 software and calculations on the distances, we obtain the D-H parameters. From the above analysis, the UAJ axis has angle $38.23^{\circ}$ with the knee axis and the STJ axis has angle $60.21^{\circ}$ with the UAJ axis.

We take $\vartheta_{d p}, \vartheta_{i e}$ as the new variables for the dorsiflexion/plantarflexion and inversion/eversion angles measured from the standing posture, and insert $\vartheta_{2}=\vartheta_{2}^{o}+\vartheta_{d p}, \vartheta_{3}=$ $\vartheta_{3}^{o}+\vartheta_{i e}$ into eqts (1). Now $\vartheta_{d p}, \vartheta_{i e}$ are the input variables of the model. Common ranges for the movement are $-40^{\circ} \leq$ $\vartheta_{d p} \leq 30^{\circ},-20^{\circ} \leq \vartheta_{i e} \leq 20^{\circ}$ [18]. Based on the model, we specify the foot workspace when inputs range through all possible motions. Our first requirement is the shank to be fixed and vertical wrt the World Coordinate System (WCS) attached to the base of the robot. All the following simulation data are referred in the WCS.

We fix point $P_{f}$ in the sole under the ankle where the center of the moving platform will be attached. We assume $P_{f}$ is on the positive axis of the knee's frame and has a distance equal to this of $P_{6}$. The workspace produced by the foot will be derived from the motion study of $P_{f}$. By eqts (1) and letting inputs $\vartheta_{d p}, \vartheta_{i e}$ run through their entire regions, $P_{f}$ traces the surface of fig. 2(a). Feet of every size and anatomy produce the surface in fig. 2(a). The geometric characteristics of this surface (e.g. shape, curvature), depend on $\alpha_{i}, a_{i}, d_{i}$. Every trajectory traced by $P_{f}$ is within this surface.

TABLE I

COORDINATE RANGES OF A SOLE-POINT UNDER THE ANKLE.

\begin{tabular}{|l|l|l|l|l|l|}
\hline \multicolumn{2}{|c|}{$\Delta \mathrm{X}=56 \mathrm{~mm}$} & \multicolumn{2}{c|}{$\Delta \mathrm{Y}=41.7 \mathrm{~mm}$} & \multicolumn{2}{c|}{$\Delta \mathrm{Z}=17.3 \mathrm{~mm}$} \\
\hline Min $\mathrm{X}$ & Max X & Min Y & Max Y & Min Z & Max Z \\
\hline-33.5 & 22.4 & -19.6 & 22 & -2.8 & 14.4 \\
\hline
\end{tabular}

We compute the orientation of the foot when its axes are rotated in specific angles. First, we establish a reference frame with its origin at $P_{f}$. The axes are parallel with those of the $O_{1}$ when the foot is in the neutral position. The rotation angles $\operatorname{roll}(\alpha), \operatorname{pitch}(\beta), \operatorname{yaw}(\gamma)$ of this frame wrt the base frame are the rotation angles of the moving platform. The foot model, when $\vartheta_{d p}, \vartheta_{i e}$ take all values in their ranges, yield the rotation workspace in fig. 2(b).

By assuming that the angle axes parameters in eqts (1) are found in well specified intervals, we will specify the extended workspace produced by the model: In [12], the orientation of 


\begin{tabular}{|l|l|l|l|l|l|}
\hline $\operatorname{Min} \alpha$ & $\operatorname{Max} \alpha$ & $\operatorname{Min} \beta$ & $\operatorname{Max} \beta$ & $\operatorname{Min} \gamma$ & $\operatorname{Max} \gamma$ \\
\hline-20.71 & 9.84 & -39.95 & 36.63 & -25.34 & 37.15 \\
\hline
\end{tabular}

the lower limp rotation axes, and the ranges in the relevant angles between them are measured. The results depend on the position of the foot even for a given patient. Different patients will give different results. We conclude that the model parameters are quite uncertain and so the model must be extended to include uncertainties. By Maximize, Minimize of Maple9.5, eqts (1) yield table III.

TABLE III

EXTENDED COORDINATE RANGES OF A SOLE-POINT UNDER THE ANKLE.

\begin{tabular}{|l|l|l|l|l|l|}
\hline \multicolumn{2}{|c|}{$\Delta \mathrm{X}=114.2 \mathrm{~mm}$} & \multicolumn{2}{c|}{$\Delta \mathrm{Y}=98.9 \mathrm{~mm}$} & \multicolumn{2}{c|}{$\Delta \mathrm{Z}=37.3 \mathrm{~mm}$} \\
\hline Min X & Max X & Min Y & Max Y & Min Z & Max Z \\
\hline-67.6 & 46.9 & -4.55 & 53.4 & -7.6 & 29.7 \\
\hline
\end{tabular}

We first used interval analysis. Supposing the parameters lie in well defined intervals, the parametric eqts (1) were expressed in interval terms. Our experiments with INTLAB confirm that the values $x_{o}, y_{o}, z_{o}$ lie in the computed intervals.

\section{A. Experimental data}

A Mephisto 3D Scanner [2] was used to take images of the right foot sole of 11 adult healthy human subjects of different age (22-42 years old), height, weight and gender. We used 5 positions: Neutral, Right-Up, Right-Down, LeftUp and Left-Down. The reference is a central point on the sole under the ankle because this point will be controlled by the platform. The coordinate differences among the point's positions define the following enclosing volume: $\Delta \mathrm{X}=98.3$ $\mathrm{mm}, \Delta \mathrm{Y}=103.5 \mathrm{~mm}, \Delta \mathrm{Z}=74.9 \mathrm{~mm}$.

To measure orientation angles, rotation velocities, and accelerations, we performed experiments with the MTi motion sensor of XSens Motion Technologies [24]. It provides and records pitch, roll and yaw angles, rate of turn and linear accelerations in axes $X, Y, Z$. We used the right foot of 5 adult healthy humans of both genders and different heights, with the shank kept vertical and fixed. The only moving part was the foot. The sensor was attached on the foot sole-point under the ankle. Data were recorded during dorsiflexionplantarflexion and inversion-eversion throughout the entire range of movement. Fig. 3 shows roll, pitch and yaw wrt time of a foot, in extreme rotational movement. Fig. 4 shows angular velocities wrt time in extreme rotational movement. The experimental $\mathrm{min} / \mathrm{max}$ values are shown in tables IV $(\operatorname{roll}(\alpha), \operatorname{pitch}(\beta)$ and $\operatorname{yaw}(\gamma)(\operatorname{deg}))$, (linear accelerations $\left.\left(\alpha_{x}, \alpha_{y}, \alpha_{z}\right)\left(\mathrm{m} / \mathrm{s}^{2}\right)\right)$ and (angular velocities $\left.\left(\omega_{x}, \omega_{y}, \omega_{z}\right)(\mathrm{rad} / \mathrm{s})\right)$.

Previous measurements of ankle muscle stength have shown that the torque producing potential of the plantraflexors is $\leq 200 \mathrm{Nm}$ [21],[16]. For the dorsiflexors and the ankle invertors and evertors the maximum torque values produced

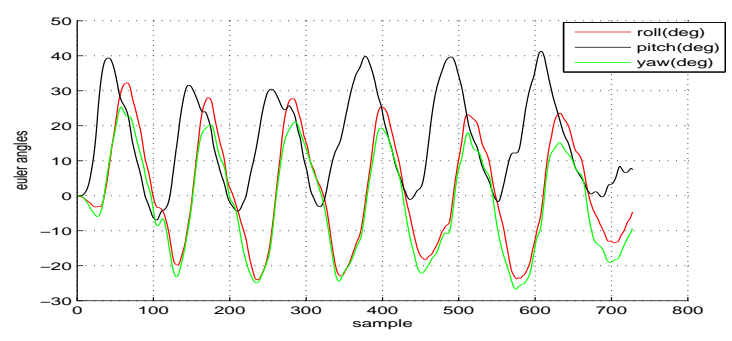

Fig. 3. Euler angles of foot on the extreme rotational motion.

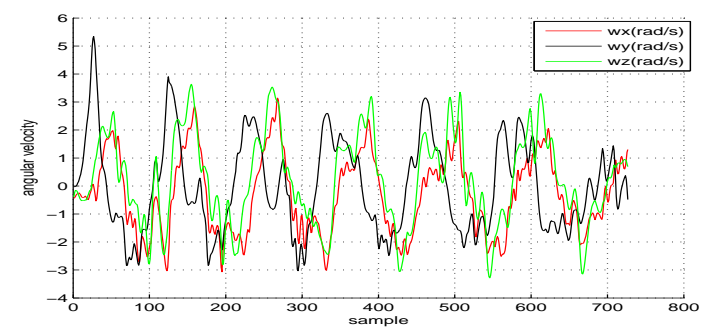

Fig. 4. Angular velocities of foot in extreme rotational motion.

during a strength test are $\leq 100 \mathrm{Nm}$ [16]. Thus, our platform will operate up to $200 \mathrm{Nm}$, to handle torque-producing tasks at different velocities during concentric or eccentric muscle actions.

Interesting results of foot study have been found in the gait analysis literature [6]. These are related with the full lower limb behavior during walking. Useful information about the ankle-foot movements were extracted from gait analysis experiments and simulation [3]. This helped us to better understand the influence of the reaction force on the lower limbs.

\section{ROBOT-BASED PHYSIOTHERAPY MECHANISMS}

For the robot design we considered existing and on-going research on robot physiotherapy [4],[22]. Wire robots are flexible, light and easier to construct, but they are not stiff nor stable enough for our purpose. An orthosis is a device applied externally to a part of the body [20]. It is used to improve function by supporting or assisting the musculoneuro-skeletal system; however, to design and develop a robotic orthosis is costly and difficult to construct.

In general, industrial serial robots provide the necessary accuracy, programming efficiency and capabilities to perform

TABLE IV

DATA RANGES BASED ON OUR EXPERIMENTS.

\begin{tabular}{|c|c|c|c|c|c|}
\hline $\operatorname{Min} \alpha$ & $\operatorname{Max} \alpha$ & $\operatorname{Min} \beta$ & $\operatorname{Max} \beta$ & Min $\gamma$ & $\operatorname{Max} \gamma$ \\
\hline-45.79 & 66.52 & -50.63 & 66.57 & -40 & 64.68 \\
\hline $\operatorname{Min} \alpha_{x}$ & $\operatorname{Max} \alpha_{x}$ & $\operatorname{Min} \alpha_{y}$ & $\operatorname{Max} \alpha_{y}$ & $\operatorname{Min} \alpha_{z}$ & $\operatorname{Max} \alpha_{z}$ \\
\hline-14.88 & 18.68 & -21.85 & 28.94 & -10.66 & 19.37 \\
\hline Min $\omega_{x}$ & $\operatorname{Max} \omega_{x}$ & Min $\omega_{y}$ & $\overline{\operatorname{Max} \omega_{y}}$ & $\operatorname{Min} \omega_{z}$ & $\overline{\operatorname{Max}} \omega_{z}$ \\
\hline-9.3 & 11.33 & $\begin{array}{l}-8.43 \\
\end{array}$ & 8.02 & $\begin{array}{l}-7.64 \\
\end{array}$ & 5.72 \\
\hline
\end{tabular}


the task [23],[22]. On the other hand, they are cumbersome and expensive for the current approach. Based on the above motion analysis, we experimented with our currently available 6-axes serial industrial robot (Earnest-X SAPRI). Its workspace is constrained in a vertical range of $240 \mathrm{~cm}$ and longitudinal range of $172 \mathrm{~cm}$, and is able to perform all required complicated movements. However, the robot is oversized for our tasks, and redundant for simpler motions. Hence, we opt for a simpler design that should satisfy our requirements of economy and ease of transportation and use.

One target should be a 3 -axes serial robot in which the rotation axes can be adapted to be collinear to the rotational axes of the ankle. This is too restrictive and overall, does not offer sufficient stiffness, rigidity and manipulability.

Parallel robots [17] are closer to our application because our workspace is confined and the robot mass to force handling ratio is much smaller. The forces that can be exerted to the robot when stepping on the device are quite high, however the torque for performing the physiotherapy exercises should be much smaller and accurately controlled. Therefore, we decompose the forces and torques on the ankle to distinguish between conditions which are acceptable according to the training session against others that can harm the ankle.

One possible solution should be the Agile-Eye 3-DOF platform [10]. Its main feature is that the moving platform rotates spherically around an external point. This should match in our case if the foot model was a spherical joint but this model is legacy. However, the most promising candidate is a properly sized tripod[15], together with an additional (and optional) rotational axis on the platform, which provides all necessary characteristics and flexibility. This is smaller and simpler than a Stewart platform, it has no redundancy, and its workspace answers all specifications obtained from our experiments.

\section{CONCLUSION}

In this work a foot model from the biomechanics literature has been adopted for the calculation of the desired workspace of robot as well as for the detailed description of foot motions. We look in detail to the ankle structure as well as in the ankle axes position e.g. the axis of rotation inversion/eversion is not parallel to the horizontal plane. Experiments and quantitative analysis using a 3D scanner and an inertial and orientation sensor provided the necessary data to specify the design features of the robotic device. Interestingly, our data are in agreement with those of the 2R foot model.

This workspace and trajectory characteristics is used for the parametric design of the geometric parameters of the aforementioned parallel tripod with an additional (optional) rotation dof, chosen for its modularity, simplicity, accuracy and generality. This device should offer networking capabilities in order to allow therapists to remotely monitor the patient's progress. The identification of foot parameters will be undertaken based on calibration approaches [8]. This is crucial since different patients have different orientation axes [12].

The functionalities of such a device are complex and the task planing in order to perform therapy is very complex. The robot-based approach must be careful and a complete study of all anatomical elements concerning the foot (muscle, tendon, ligament, articulation) will be taken into account in the operation. This will avoid the damage of the anatomical structures.

We are investigating simulation with Anybody [3] software to assist in this effort working on Musculoskeletal Simulation of Rehabilitation Program approach [14]. The final step is to develop a prototype; experiments on patients will be extensively carried out under the supervision of physiotherapists.

\section{REFERENCES}

[1] http://laimuz.unizar.es/teledoc/home.html.

[2] http://www.thinglab.co.uk

[3] ANYBODY. http://www.anybodytech.com.

[4] M. Armada. www.iai.csic.es/iarp/sapr $/ 12_{s}$ pain $_{i} \operatorname{arp}_{j} c f_{2}$ 003.pdf. International Advanced Robotics Programme (IARP) 22nd JOINT COORDINATING FORUM, pages 347-352, 2003.

[5] G. Burdea, M. Girone, M. Bouzit, V. Popescu, and J.E Deutsch. A Stewart platform-based system for ankle telerehabilitation. $A u$ tonomous Robots, 10:203-212, 2001.

[6] ClinicalGaitAnalysis. http://www.univie.ac.at/cga/

[7] J.S. Dai, T. Zhao, and C. Nester. Sprained ankle physiotherapy based mechanism synthesis and stiffness analysis of a robotic rehabilitation device. Autonomous Robots, 16:207-218, 2004.

[8] D.Daney and I.Z Emiris. Robust parellel robot calibration with partial information. Proc. IEEE Int. Conf. Robotics and Automation, pages 3262-3267, 2001. Seoul, S. Korea.

[9] J. Dul and G.E Johnson. A kinematic model of the human ankle. $J$. of Biomedical Engineering, 7:137-143, 1985.

[10] C.M. Gosselin and J. Wang. Singularity Loci of a Special Class of Sphericsl Three-degree-of-freedom Parallel Mechanisms with Revolute Actuators. Int. J. of Robotics Research, 21(7) pp.649-659, 2002.

[11] F. Hao and J-P. Merlet. Multi-criteria optimal design of parallel manipulators based on interval analysis. J. Mechanism and Machine Theory, 40(2):pp.157-171, 2005.

[12] R.E. Isman and V.T Inman. Anthropometric studies of the human foot and ankle. Bulletin of Prosthetic Research, 10-11:97-129, 1969.

[13] L. Jaulin, M. Kieffer, O. Didrit, and E. Walter. Appl. Interval Analysis. Springer-Verlag, 2001.

[14] L.F Lee and V.N Krovi. Musculoskeletal simulation based optimization of rehabilitation program. Virtual Rehabilitation, pages 36-41, 2006.

[15] K. Lee, D.K. Shah Kinematic Analysis of a tree degrees of freedom in parallel actuated manipulator. IEEE J. of Rob. and Automation, Vol 4(3) pages 354-60, 2003.

[16] C.N. Maganaris, V. Baltzopoulos, D. Ball, and A.J. Sargeant. In vivo specific tension of human skeletal muscle. J. Appl. Physiol., 90(3):865-72, 2001.

[17] J-P. Merlet. Parallel Robots. Springer-Verlag, Second edition, 2006.

[18] B.M Nigg, V.Fisher, T.L. Allinger, J.R. Ronsky, and J.R. Engsberg. Range of motion of the foot as a function of age. Foot and Ankle, 16(6):336-343, 1992.

[19] PerformBetter. http://www.performbetter.com.

[20] RCAI. http://www.rcai.com.

[21] D. Sale, J. Quinlan, E. Marsh, A.J. McComas , and A.Y. Belanger. Influence of joint position on ankle plantarflexion in humans. J. Appl. Physiol., 52(6):1636-42, 1982.

[22] J. Savage M. Levesley R. Richardson A. Cozens M.M Williams B. Bhakta P. Culmer, A. Jackson. From single to dual robotic therapy: A review of the development process of iPAM. Biomed. Robotics and Biomechatronics, pages 347-352, 2003.

[23] A. Toth and I. Ermolaev. Robots with patients. Engineer IT, pages 60-62, 2006.

[24] XSens Motion Technologies. http://www.xsens.com 